\title{
SET-VALUED MEASURES
}

\author{
BY \\ ZVI ARTSTEIN
}

\begin{abstract}
A set-valued measure is a $\sigma$-additive set-function which takes on values in the nonempty subsets of a euclidean space. It is shown that a bounded and nonatomic set-valued measure has convex values. Also the existence of selectors (vectorvalued measures) is investigated. The Radon-Nikodym derivative of a set-valued measure is a set-valued function. A general theorem on the existence of R.-N. derivatives is established. The techniques require investigations of measurable setvalued functions and their support functions.
\end{abstract}

1. Introduction. Let $(T, \mathscr{T})$ be a measurable space, and $S$ be a finite-dimensional real vector space. A set-valued measure $\Phi$ on $(T, \mathscr{T})$ is a function from $T$ to the nonempty subsets of $S$, which is countably additive, i.e. $\Phi\left(\bigcup_{j=1}^{\infty} E_{j}\right)=\sum_{j=1}^{\infty} \Phi\left(E_{j}\right)$ for every sequence $E_{1}, E_{2}, \ldots$, of mutually disjoint elements of $\mathscr{T}$. Here the sum $\sum_{j=1}^{\infty} A_{j}$ of the subsets $A_{1}, A_{2}, \ldots$, of $S$, consists of all the vectors $a=\sum_{j=1}^{\infty} a_{j}$, where the series is absolutely convergent, and $a_{j} \in A$, for every $j=1,2, \ldots$.

The calculus of set-valued functions has recently been developed by several authors. (See Aumann [1], Banks and Jacobs [3], Bridgland [4], Debreu [7], Hermes [11], Hukuhara [15], and Jacobs [16].) The ideas and techniques have many interesting applications in mathematical economics, [2], [7], [13]; in control theory, [11], [12], [16]; and in other mathematical fields (see [3] and [4] for extensive lists of references). Also, set-valued measures have been discussed in connection with applications and for their own interest. See Debreu and Schmeidler [8], Schmeidler [19], and Vind [20]. It is our purpose in this paper to extend the basic theory of set-valued measures. We shall take special interest in the following three subjects: convexity of bounded set-valued measures, existence of selectors, the Radon-Nikodym derivative of a set-valued measure.

The main results in the three branches are as follows.

The values of a nonatomic and bounded set-valued measure are convex sets. (I found this question in Schmeidler's paper [19]. Schmeidler proved the theorem under the additional assumption that $\Phi(T)$ is compact.)

If $\Phi$ is a set-valued measure with convex values, which is absolutely continuous with respect to a finite measure, then for every $E \in \mathscr{T}$ and $x \in \Phi(E)$, there exists a

Received by the editors May 5, 1971.

AMS 1970 subject classifications. Primary 28-00, 28A55; Secondary 26A51.

Key words and phrases. Set-valued measure, measurable set-valued function, support function, integral of a set-valued function, selectors, Lyapunov's convexity theorem, RadonNikodym derivatives. 
selector $\mu$ of $\Phi$ such that $\mu(E)=x$. We show that none of the conditions can be removed.

The Radon-Nikodym derivative of a set-valued measure is a set-valued function, while the integration of the latter is in the sense of Aumann [1]. Following Debreu and Schmeidler [8], we investigate those set-valued measures which have derivatives with convex and closed values, and respectively, with convex and relatively open values. Our contribution is to set the results for set-valued measures which might have values not only in the positive orthant. We also give counterexamples when the result in [8] cannot be generalized. Finally, we introduce a general theorem on the existence of derivatives. In this theorem we use the Continuum Hypothesis.

In order to obtain the results we have to develop some techniques concerning integration and differentiation of the support function of a set-valued function and a set-valued measure. These results are formulated as lemmas.

The article is organized as follows. In $\$ 2$ we give notations and conventions which will be used throughout the paper. Each of the other sections begins with more definitions and notations which are needed there. In $\$ 4$ we deal with bounded set-valued measures. $\S \S 5$ to 7 are the sections of the lemmas. Their subjects are measurable set-valued functions, support functions, and integration of set-valued functions respectively. In $\S 8$ the existence and properties of selectors are investigated. The Radon-Nikodym derivatives are treated in the two final sections.

2. Notations and conventions. The empty set is denoted by $\varnothing$. The euclidean norm on $S$ is \|\| . The scalar product of two elements $p$ and $x$ of $S$ is denoted by $p \cdot x$. We do not distinguish between $S$ and its dual, but we try to use the letters $p$ and $q$ for dual vectors, while $x$ and $y$ are elements of $S$. If $A$ is a subset of $S$ then cl $A$ is the closure of $A$, and co $A$ is the convex hull of $A$. If $A$ is convex then ri $A$ is the relative interior of $A$. For two sets $A$ and $B$, and a real number $\alpha$ we denote $A+B=\{a+b: a \in A, b \in B\}$, and $\alpha A=\{\alpha a: a \in A\}$.

A measurable space $(T, \mathscr{T})$ consists of a set $T$, and a $\sigma$-field $\mathscr{T}$ of the "measurable" subsets of $T$. No topological structure on $T$ is required. By a measure on $(T, \mathscr{T})$ we mean a real signed measure which might have $+\infty$ as a value. We shall speak on finite measures and on nonnegative measures. If $\lambda$ is a measure then $|\lambda|(E)$ denotes the total variation of $\lambda$ over $E$. In particular $|\lambda|$ is a nonnegative measure. The measure $\nu$ is absolutely continuous with respect to $\lambda$ if $|\lambda|(E)=0$ implies $\nu(E)=0$. We denote this by $\nu \ll \lambda$. An atom of the measure $\lambda$ is a set $E$, with $|\lambda|(E)>0$, such that if $E_{1} \subset E$ then either $\lambda\left(E_{1}\right)=0$ or $\lambda\left(E \backslash E_{1}\right)=0$. A measure with no atoms is nonatomic.

3. Two basic properties. Let $\Phi$ be a set-valued measure on $(T, \mathscr{T})$ and let $p \in S$. For every $E \in \mathscr{T}$ denote $\nu_{p}(E)=\sup \{p \cdot x: x \in \Phi(E)\}$, and $\Phi_{p}(E)$ $=\left\{x \in \Phi(E): p \cdot x=v_{p}(E)\right\}$. (The set-function $\nu_{p}$ depends, of course, on the setvalued measure $\Phi$.) 
The two propositions below are well known and have appeared in the literature in several forms. We state them here in a form suitable for the sequel, and for completeness we also give the proofs.

Proposition 3.1. The set function $\nu_{p}(\cdot)$ is a measure.

Proof. The set function $\nu_{p}(\cdot)$ is well defined and has values in $(-\infty, \infty]$. It is clear that $\nu_{p}(\cdot)$ is finitely additive. We have to show that if $E_{j}(j=1,2, \ldots)$ is a sequence of disjoint elements of $\mathscr{T}$, and if $E=\bigcup_{j=1}^{\infty} E_{j}$, then $\nu_{p}(E)=\sum_{j=1}^{\infty} \nu_{p}\left(E_{j}\right)$.

If $x \in \Phi(E)$ then $x=\sum_{j=1}^{\infty} x_{j}$, where $x_{j} \in \Phi\left(E_{j}\right)$ for $j=1,2, \ldots$ Then $p \cdot x$ $=\sum_{j=1}^{\infty} p \cdot x_{j}$ and this implies $\nu_{p}(E) \leqq \lim \inf _{k} \sum_{j=1}^{k} \nu_{p}\left(E_{j}\right)$. If $\nu_{p}(E)=\infty$ there is nothing else to show. If $\nu_{p}(E)<\infty$, the additivity implies $\nu_{p}\left(E_{j}\right)<\infty$ for every $j$. Given $\varepsilon>0$, choose, for each $j$, an element $y_{j} \in \Phi\left(E_{j}\right)$ such that $\nu_{p}\left(E_{j}\right)-p \cdot y_{j}<2^{-j} \varepsilon$. Denote $z^{m}=y_{1}+\cdots+y_{m}+\sum_{j>m} x_{j}$. Then $z^{m} \in \Phi(E)$ and

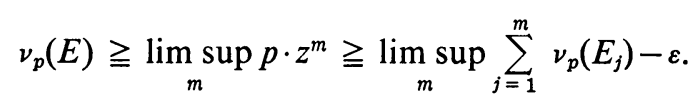

Since $\varepsilon$ is arbitrarily small, this implies $\nu_{p}(E) \geqq \lim \sup _{m} \sum_{j=1}^{m} \nu_{p}\left(E_{j}\right)$. Q.E.D.

Proposition 3.2. The set-valued set-function $\Phi_{p}$ is countably additive.

Proof. We do not claim that $\Phi_{p}$ is a set-valued measure. Indeed, $\Phi_{p}(E)$ might be empty. Let $E_{j}(j=1,2, \ldots)$ be a sequence of mutually disjoint elements in $\mathscr{T}$, and let $E=\bigcup_{j=1}^{\infty} E_{j}$. It is clear that $\sum_{j=1}^{\infty} \Phi_{p}\left(E_{j}\right)$ is included in $\Phi_{p}(E)$. Conversely, if $x \in \Phi_{p}(E)$ then $x=\sum_{j=1}^{\infty} x_{j}$ where $x_{j} \in \Phi\left(E_{j}\right)$. Notice that

$$
p \cdot x=\sum_{j=1}^{\infty} p \cdot x_{j} \leqq \sum_{j=1}^{\infty} \nu_{p}\left(E_{j}\right)=\nu_{p}(E)=p \cdot x
$$

and since $p \cdot x_{j} \leqq v_{p}\left(E_{j}\right)$ for each $j$ this implies $p \cdot x_{j}=v_{p}\left(E_{j}\right)$ for each $j$. Thus $\Phi_{p}(E)$ is included in $\sum_{j=1}^{\infty} \Phi_{p}\left(E_{j}\right)$. Q.E.D.

4. Bounded set-valued measures. A set-valued measure $\Phi$ is bounded if $\Phi(T)$ is a bounded set. The additivity implies that $\Phi(T)$ includes a translation of every $\Phi(E)$, thus $\Phi$ is bounded if and only if $\Phi(E)$ is bounded for every $E \in \mathscr{T}$. The additivity of $\Phi$ also implies that either $\Phi(\varnothing)=\{0\}$ or $\Phi(\varnothing)$ is an unbounded set. Thus $\Phi$ is bounded implies $\Phi(\varnothing)=\{0\}$.

If $\Phi$ is a bounded set-valued measure then for every $p$ in $S$ the measure $\nu_{p}$ is finite. Let $n$ be the dimension of $S$ and let $e_{1}, \ldots, e_{2 n}$ be the $2 n$ vectors $(0, \ldots, \pm 1, \ldots, 0)$. Denote $\nu=\sum_{i=1}^{2 n}\left|\nu_{e_{i}}\right|$. Then $\nu$ is a finite and nonnegative measure on $(T, \mathscr{T})$. Also $\nu(E)=0$ if and only if $\Phi(E)=\{0\}$. Notice that $x \in \Phi(E)$ implies $\|x\| \leqq \nu(E) \leqq \nu(T)$. Therefore: If $\Phi$ is a bounded set-valued measure, then the range of $\Phi$, i.e. $\bigcup_{E \in \mathscr{T}} \Phi(E)$, is a bounded set.

An atom of the set-valued measure $\Phi$ is an element $E \in \mathscr{T}$, for which $\Phi(E) \neq\{0\}$, and such that if $E_{1} \subset E$ then either $\Phi\left(E_{1}\right)=\{0\}$ or $\Phi\left(E \backslash E_{1}\right)=\{0\}$. A set-valued measure with no atoms is nonatomic. Notice that $\Phi$ is nonatomic if and only if $\nu$ is nonatomic. 
LEMMA 4.1. Let I be the unit interval. Let $\lambda_{1}, \ldots, \lambda_{n}$ be nonatomic finite measures on I. Denote $\lambda=\sum_{i=1}^{n}\left|\lambda_{i}\right|$. Then for every real number $\theta$, satisfying $0<\theta<1$, there exists a subset $I_{\theta}$ of $I$ such that

(a) $\lambda_{i}\left(I_{\theta}\right)=\theta \lambda_{i}(I)$ for $i=1, \ldots, n$.

(b) Each $I_{\theta}$ and $I \backslash I_{\theta}$ differs from a denumerable union of intervals only by a set of $\lambda$-measure zero.

Proof. Theorem $1^{*}$ of Halkin [9] implies that one can choose $I_{\theta}$ to be even a finite union of intervals. Anyway, we offer here a short proof of the above weaker version. Without loss of generality $\lambda_{i} \ll \lambda_{1}$ for $i=1,2, \ldots, n$. Indeed, write $\lambda_{0}$ $=\sum_{i=1}^{n}\left|\lambda_{i}\right|$ and prove the lemma for $\lambda_{0}, \lambda_{1}, \ldots, \lambda_{n}$. The case $n=1$ is trivial. Proceed by induction and add to the induction hypothesis the requirement $\theta<\zeta \Rightarrow I_{\theta} \subset I_{\zeta}$. Let $J_{\theta}$ for $0 \leqq \theta \leqq 1$ be an appropriate family for the measures $\lambda_{1}, \ldots, \lambda_{n-1}$. Define $I(\zeta)=\left(J_{1 / 2} \mid J_{\zeta}\right) \cup\left(I \backslash J_{1-\zeta}\right)$, for $0 \leqq \zeta \leqq \frac{1}{2}$. Then $\lambda_{i}(I(\zeta))=\frac{1}{2} \lambda_{i}(I)$ for each $\zeta$, and $i=1, \ldots, n-1$. Also $\lambda_{n}(I(\zeta))$ is continuous with respect to $\zeta$. This implies the existence of a certain $\zeta_{1}$ for which $\lambda_{n}\left(I\left(\zeta_{1}\right)\right)=\frac{1}{2} \lambda_{n}(I)$. Denote $I_{1 / 2}=I\left(\zeta_{1}\right)$. Each $I_{1 / 2}$ and $I \backslash I_{1 / 2}$ is essentially a countable union of intervals. Applying the above procedure to each of these intervals will lead to a construction of $I_{1 / 4}$ and $I_{3 / 4}$ out of $I_{1 / 2}$ and $I \backslash I_{1 / 2}$, and for $\theta=\frac{1}{4}, \frac{1}{2}, \frac{3}{4}$ the requirements (a) and (b) hold. Generally, this procedure will lead to construction of $I_{\eta}$ for every dyadic $\eta$, such that (a), (b) and the inclusion requirement still hold. Finally, define $I_{\theta}$ to be the union of all $I_{n}$ such that $\eta$ is dyadic, and $\eta \leqq \theta$. This completes the induction step. Q.E.D.

Let $E$ be an element of $\mathscr{T}$. A dyadic structure of $E$ is a collection of measurable sets $\left\langle E\left(\varepsilon_{1} \varepsilon_{2} \cdots \varepsilon_{k}\right)\right\rangle$, where $\varepsilon_{i}=0,1$ and $k=1,2, \ldots$, such that

(a) $E\left(\varepsilon_{1} \cdots \varepsilon_{k} 0\right) \cup E\left(\varepsilon_{1} \cdots \varepsilon_{k} 1\right)=E\left(\varepsilon_{1} \cdots \varepsilon_{k}\right)$ and $E(0) \cup E(1)=E$.

(b) $E\left(\varepsilon_{1} \cdots \varepsilon_{k} 0\right) \cap E\left(\varepsilon_{1} \cdots \varepsilon_{k} 1\right)=\varnothing$ and $E(0) \cap E(1)=\varnothing$.

The standard dyadic structure of the unit interval $I=[0,1)$ is the collection $\left\langle I\left(\varepsilon_{1} \varepsilon_{2} \cdots \varepsilon_{k}\right)\right\rangle$ described by $I(0)=\left[0, \frac{1}{2}\right), I(00)=\left[0, \frac{1}{4}\right), I(01)=\left[\frac{1}{4}, \frac{1}{2}\right)$, and generally

$$
I\left(\varepsilon_{1} \cdots \varepsilon_{k}\right)=\left[\sum_{i=1}^{k} \varepsilon_{i} 2^{-i}, 2^{-k}+\sum_{i=1}^{k} \varepsilon_{i} 2^{-i}\right) .
$$

THEOREM 4.2. If $\Phi$ is a bounded, nonatomic set-valued measure, then for every $E \in \mathscr{T}$, the set $\Phi(E)$ is convex.

Proof. Let $x$ and $y$ be two vectors in $\Phi(E)$ and let $\theta$ be a real number, $0<\theta<1$. We show that $\theta x+(1-\theta) y \in \Phi(E)$. Define $\nu$ as above. Since $v$ is nonatomic there exists a dyadic structure $\left\langle E\left(\varepsilon_{1} \cdots \varepsilon_{k}\right)\right\rangle$ of $E$ such that $\nu\left(E\left(\varepsilon_{1} \cdots \varepsilon_{k}\right)\right)=2^{-k} \nu(E)$. The additivity of $\Phi$ implies the existence of vectors $x\left(\varepsilon_{1} \cdots \varepsilon_{k}\right), y\left(\varepsilon_{1} \cdots \varepsilon_{k}\right)$ for $\varepsilon_{i}=0,1$ and $k=1,2, \ldots$, such that

(a) $x\left(\varepsilon_{1} \cdots \varepsilon_{k}\right)$ and $y\left(\varepsilon_{1} \cdots \varepsilon_{k}\right)$ belong to $\Phi\left(E\left(\varepsilon_{1} \cdots \varepsilon_{k}\right)\right)$.

(b) $x\left(\varepsilon_{1} \cdots \varepsilon_{k} 0\right)+x\left(\varepsilon_{1} \cdots \varepsilon_{k} 1\right)=x\left(\varepsilon_{1} \cdots \varepsilon_{k}\right)$ and $y\left(\varepsilon_{1} \cdots \varepsilon_{k} 0\right)+y\left(\varepsilon_{1} \cdots \varepsilon_{k} 1\right)=$ $y\left(\varepsilon_{1} \cdots \varepsilon_{k}\right)$.

(c) $x(0)+x(1)=x$ and $y(0)+y(1)=y$. 
Define $\boldsymbol{x}\left(E\left(\varepsilon_{1} \cdots \varepsilon_{k}\right)\right)=x\left(\varepsilon_{1} \cdots \varepsilon_{k}\right)$ and $\boldsymbol{y}\left(E\left(\varepsilon_{1} \cdots \varepsilon_{k}\right)\right)=y\left(\varepsilon_{1} \cdots \varepsilon_{k}\right)$. Then $\boldsymbol{x}(\cdot)$ and $\boldsymbol{y}(\cdot)$ are additive vector-valued set-functions on the dyadic structure. Since $\|\boldsymbol{x}(\cdot)\|$ and $\|\boldsymbol{y}(\cdot)\|$ are dominated by $\nu(\cdot)$, the set functions $\boldsymbol{x}$ and $\boldsymbol{y}$ are countably additive, and have extensions to finite vector measures on the $\sigma$-field $\mathscr{T}_{1}$, which is generated by the dyadic structure. Denote these extensions by the same symbols $\boldsymbol{x}$ and $\boldsymbol{y}$. Since $v$ is nonatomic on $\mathscr{T}_{1}$, the measures $\boldsymbol{x}$ and $\boldsymbol{y}$ are nonatomic.

Consider the $(2 n+1)$-dimensional vector-valued measure $(\nu, \boldsymbol{x}, \boldsymbol{y})$, defined on $\mathscr{T}_{1}$. In view of Lemma 4.1 and the canonic isomorphism between the dyadic structure $\left\langle E\left(\varepsilon_{1} \cdots \varepsilon_{k}\right)\right\rangle$ and the standard dyadic structure $\left\langle I\left(\varepsilon_{1} \cdots \varepsilon_{k}\right)\right\rangle$ of the unit interval, there exists an $E_{1} \subset E$ such that

(d) $\boldsymbol{x}\left(E_{1}\right)=\theta \boldsymbol{x}(E)$ and $\boldsymbol{y}\left(E_{1}\right)=\theta \boldsymbol{y}(E)$.

(e) Each $E_{1}$ and $E \backslash E_{1}$ differs only by a $\nu$-null set from a denumerable union of dyadic elements.

Notice that if $G$ is a denumerable union of dyadic elements then $x(G)$ and $y(G)$ are members of $\Phi(G)$. This follows from the $\sigma$-additivity of $\Phi$ and from condition (a) in the beginning of the proof. If $G^{\prime}$ differs only by a $\nu$-null set from $G$, then $\Phi(G)=\Phi\left(G^{\prime}\right)$. This follows from $v(H)=0$ if and only if $\Phi(H)=\{0\}$.

The last remark implies that $\theta x=\boldsymbol{x}\left(E_{1}\right) \in \Phi\left(E_{1}\right)$ and $(1-\theta) y=y\left(E \backslash E_{1}\right) \in \Phi\left(E \backslash E_{1}\right)$. Finally, $\theta x+(1-\theta) y \in \Phi\left(E_{1}\right)+\Phi\left(E \backslash E_{1}\right)=\Phi(E)$. Q.E.D.

COROLlaRY 4.3. Under the conditions of Theorem 4.2, let $x \in \Phi(E)$ and $0<\theta<1$, then there exists an $E_{1} \subset E$ such that $\theta x \in \Phi\left(E_{1}\right)$.

Proof. We have already proved this while proving Theorem 4.2. Notice that the corollary is implied by (d) and (e) and the remark following them.

REMARK. Lemma 4.1 contains a stronger version of the Lyapunov convexity theorem (see Halmos [10]). However, the convexity theorem can be easily derived from Theorem 4.2 as follows. Let $\mu$ be a nonatomic vector measure on $(T, \mathscr{T})$. Denote by $\Phi(E)$ the range of $\mu \mid E$. It is easy to verify that $\Phi$ is a nonatomic bounded set-valued measure. Since Theorem 4.2 implies that $\Phi$ has convex values, the range of $\mu$, which is actually $\Phi(T)$, is convex.

THEOREM 4.4. If $\Phi$ is a bounded nonatomic set-valued measure, then the range of $\Phi$, i.e. $\bigcup_{E \in \mathscr{T}} \Phi(E)$, is a convex set.

Proof. Let $x \in \Phi\left(E_{1}\right)$ and $y \in \Phi\left(E_{2}\right)$ and $0<\theta<1$. Denote $G=E_{1} \cap E_{2}$. Then $x=x_{1}+x_{2}$ and $y=y_{1}+y_{2}$ where $x_{1}$ and $y_{1}$ are elements of $\Phi(G), x_{2} \in \Phi\left(E_{1} \mid G\right)$ and $y_{2} \in \Phi\left(E_{2} \mid G\right)$. Theorem 4.2 implies that $\theta x_{1}+(1-\theta) y_{1} \in \Phi(G)$. Corollary 4.3 implies the existence of $G_{1} \subset E_{1} \mid G$ and $G_{2} \subset E_{2} \mid G$ such that $\theta x_{2} \in \Phi\left(G_{1}\right)$ and $(1-\theta) y_{2} \in \Phi\left(G_{2}\right)$. The additivity implies that $\theta x+(1-\theta) y$ is an element of $\Phi\left(G \cup G_{1} \cup G_{2}\right)$. Q.E.D.

REMARKS. The boundedness of $\Phi$ in Theorems 4.2 and 4.4 cannot be removed. We quote here an example due to W. Hildenbrand. Let $\mathscr{B}$ be the Borel $\sigma$-field of the unit interval. For each $E \in \mathscr{B}$ with a positive Lebesgue measure, the set $\Phi(E)$ is the set of all the nonnegative integers. If $E$ is of measure zero then $\Phi(E)=\{0\}$. 
The nonatomicity cannot be removed either. Indeed, let $T$ be a singleton and define $\Phi(T)=\{0,1\}$.

The sum of two nonempty convex sets is compact if and only if both sets are compact. Hence, if $\Phi$ is a bounded nonatomic set-valued measure, and $\Phi(T)$ is compact, then $\Phi(E)$ is compact for every $E \in \mathscr{T}$. Indeed, apply the equality $\Phi(E)+\Phi(T \backslash E)=\Phi(T)$ together with Theorem 4.2. See Schmeidler [19, 4.2] for a proof that in this case the range of $\Phi$ is also a compact set.

The following two propositions deal with operations on bounded set-valued measures. Detailed proofs are omitted, since it is easy to construct them from the hints.

Proposition 4.5. Let $\Phi$ be a bounded set-valued measure. Define $\bar{\Phi}$ by $\bar{\Phi}(E)$ $=\operatorname{cl} \Phi(E)$. Then $\bar{\Phi}$ is countably additive.

Hint. If $E=\bigcup_{j=1}^{\infty} E_{j}$ is a disjoint union, then every series $x=\sum_{j=1}^{\infty} x_{j}$, where $x_{j} \in \Phi\left(E_{j}\right)$, is dominated by the convergent series $\sum_{j=1}^{\infty} \nu\left(E_{j}\right)$.

Proposition 4.6. Let $\Phi$ be a bounded set-valued measure. Define $\Phi^{*}$ by $\Phi^{*}(E)$ $=\operatorname{co} \Phi(E)$. Then $\Phi^{*}$ is countably additive.

Hint. It is clear the $\Phi^{*}$ is finitely additive. In this case, this implies the countable additivity.

Both Propositions 4.5 and 4.6 are not valid without the boundedness of $\Phi$. However, Proposition 4.6 still holds if $\Phi$ takes on values in the positive orthant of $S$. See Debreu and Schmeidler [8, Lemma 5].

As a final remark of this section let us refer to Theorem 8.3 , which deals also with bounded set-valued measures.

5. Lemmas concerning measurable set-valued functions. Let $(T, \mathscr{T})$ be a measurable space. A set-valued function $F$, from $T$ to the subsets of $S$, is measurable if for every closed subset $C$ of $S$ the set

$$
F^{-1}(C)=\{t \in T: F(t) \cap C \neq \varnothing\}
$$

is a measurable subset of $T$. Note that, for $F$ to be measurable, it suffices that $F^{-1}(C)$ is measurable for every compact $C$ in $S$. Indeed, every closed set is a countable union of compact sets, and the operation $F^{-1}$ has the property $F^{-1}\left(\bigcup_{j=1}^{\infty} C_{j}\right)=\bigcup_{j=1}^{\infty} F^{-1}\left(C_{j}\right)$. Notice also that a point-valued function $g$ is measurable if and only if the set-valued function $G(t)=\{g(t)\}$ is measurable.

We do not assume that a set-valued function has only nonempty values. In particular, if $F$ is measurable, then the set $\{t: F(t) \neq \varnothing\}=F^{-1}(S)$ is measurable.

The lemmas below will be used in the sequel. They are not given in the most general form, since we prefer here a simple proof of a special case to general results with complicated proofs. 
Lemma 5.1. Let $F$ be a measurable set-valued function, and let $p$ be in $S$. Denote $s(p, t)=\sup \{p \cdot x: x \in F(t)\}(\sup \varnothing=-\infty)$. Then $s(p, \cdot)$ is a measurable function.

Proof. Let $r$ be a real number. Denote $C_{k}$ the closed subset of $S$ defined by $\{x: p \cdot x \geqq r+1 / k\}$, this for $k=1,2, \ldots$ Note that $s(p, t)>r$ if and only if $F(t) \cap C_{k}$ $\neq \varnothing$ for some $k$. Thus, the set

$$
\{t: s(p, t)>r\}=\bigcup_{k=1}^{\infty}\left\{t: F(t) \cap C_{k} \neq \varnothing\right\}
$$

is measurable. Q.E.D.

REMARK. Some authors call a set-valued function measurable if its graph is a measurable set in the product $\sigma$-field. See [1] and [8]. The definition which we adopt here enables us to prove Lemma 5.1 without $T$ being a complete $\sigma$-field. Compare with [7, (4.5)] and with [8, Lemma 1]. Debreu proved (see [18, Theorem 2]) that a measurable set-valued function with closed values has a measurable graph.

Lemma 5.2. Let $F$ be a measurable set-valued function. Define $\bar{F}$ by $\bar{F}(t)=\mathrm{cl} F(t)$. Then $\bar{F}$ is measurable.

Proof. Let $C$ be a compact subset of $S$. Let $C_{k}=C+(1 / k) B$, where $B$ is the closed unit ball of $S$. Then $\bar{F}(t) \cap C \neq \varnothing$ if and only if $F(t) \cap C_{k} \neq \varnothing$ for every $k=1,2, \ldots$ Thus the set

$$
\{t: \bar{F}(t) \cap C \neq \varnothing\}=\bigcap_{k=1}^{\infty}\left\{t: F(t) \cap C_{k} \neq \varnothing\right\}
$$

is measurable. Q.E.D.

LEMMA 5.3. Let $F_{j}(j=1,2, \ldots)$ be a finite or countable family of measurable set-valued functions, such that each $F_{j}$ takes on only closed values. Then the setvalued function $F(t)=\bigcap_{j} F_{j}(t)$ is measurable.

Proof. See Rockafellar [18, Corollary 1.3].

LEMMA 5.4. Let $F$ be a measurable set-valued function with closed values, and let $g$ be a measurable point valued function. Then the set $\{t: g(t) \in F(t)\}$ is measurable.

Proof. Denote $G(t)=\{g(t)\}$. As was noted above, $G$ is measurable. In view of Lemma 5.3 the set-valued function $H(t)=G(t) \cap F(t)$ is measurable. Finally, the equality $\{t: g(t) \in F(t)\}=\{t: H(t) \neq \varnothing\}$ completes the proof.

LEMMA 5.5. Let $\left\{p_{k}\right\}_{k=1}^{\infty}$ be a sequence of vectors in $S$. For every $k$ let $s(k, t)$ be a measurable real-valued function. Then:

(a) For each $k$ the set-valued function $F(t)=\{x: p \cdot x \leqq s(k, t)\}$ is measurable.

(b) For each $k$ the set-valued function $G(t)=\{x: p \cdot x=s(k, t)\}$ is measurable.

(c) The set-valued function $H(t)=\bigcap_{k=1}^{\infty}\{x: p \cdot x \leqq s(k, t)\}$ is measurable. 
Proof. Let $C$ be a compact subset of $S$. Denote $r=\min \{p \cdot x: x \in C\}$. The set $\{t: F(t) \cap C \neq \varnothing\}=\{t: s(k, t) \geqq r\}$ is obviously measurable. This completes the proof of (a). Part (a) together with Lemma 5.3 imply parts (b) and (c) as follows. Note that $G(t)=\{x: p \cdot x \leqq s(k, t)\} \cap\{x: p \cdot x \geqq s(k, t)\}$. Thus $G(t)$ is the intersection of two measurable set-valued functions with closed values. On the other hand, $H(t)$ is the intersection of a countable family of measurable set-valued functions with closed values. Q.E.D.

LEMMA 5.6. Let $F$ be a measurable set-valued function with nonempty, closed and convex values. Then the set-valued function $\mathrm{ri} F(\mathrm{ri} F(t)=\mathrm{ri}(F(t)))$ has a measurable selection, i.e. there is a measurable point-valued function $f$ such that $f(t) \in F(t)$ for every $t \in T$.

Proof. We first prove the lemma for set-valued functions with bounded values. Let $e_{1}, \ldots, e_{n}$ be the $n$ vectors $(0, \ldots, 1, \ldots, 0)$. Define the set-valued functions $F_{0}, F_{1}, \ldots, F_{n}$ as follows. Define $F_{0}(t)=F(t)$. For $i=1, \ldots, n$ let

$$
s_{i}(t)=\sup \left\{e_{i} \cdot x: x \in F_{i-1}(t)\right\}+\inf \left\{e_{i} \cdot x: x \in F_{i-1}(t)\right\}
$$

and define $F_{i}(t)=\left\{x \in F_{i-1}(t): e_{i} \cdot x=\frac{1}{2} s_{i}(t)\right\}$. If $F_{i-1}$ is measurable then $s_{i}(t)$ is a measurable function (Lemma 5.1), and thus $F_{i}$ is a measurable function (Lemma 5.5(b)). If $F_{i-1}$ has nonempty, closed, and convex values, so does $F_{i}$, and ri $F_{i}$ $\subset$ ri $F_{i-1}$. Since $F_{0}=F$ is a measurable set-valued function, with nonempty, closed, and convex value, it follows that $F_{n}$ is measurable and ri $F_{n}(t) \subset$ ri $F(t)$. Finally, notice that $F_{n}(t)=\{f(t)\}$ is a singleton, for every $t$. Thus, $f(t)$ is the required selection.

The next step is to prove the lemma in the general case. Let $D_{1}, D_{2}, \ldots$ be a sequence of open subsets of $S$, whose union is $S$. Every open set is a denumberable union of compact sets, thus $T_{k}=F^{-1}\left(D_{k}\right)$ is a measurable subset of $T$. If $t \in T_{k}$ then ri $\left(F(t) \cap \mathrm{cl} D_{k}\right) \subset$ ri $F(t)$. Apply now the first part of the proof to the set-valued function $F(t) \cap \mathrm{cl} D_{k}$, restricted to $T_{k} \mid \bigcup_{j<k} T_{j}$, in order to get an appropriate selection $f(t)$ for $t \in T_{k} \mid \bigcup_{j<k} T_{j}$. This for $k=1,2, \ldots$. Finally, $t \rightarrow f(t)$ is the required selection. Q.E.D.

LEMMA 5.7. If $F$ is a measurable set-valued function with closed and convex values, then ri $F$ is also measurable.

Proof. Let $f$ be a measurable selection of ri $F$. Define

$$
F_{k}(t)=(1-1 / k)(F(t)-f(t))+f(t)
$$

It is easy to verify that each $F_{k}$ is measurable, and since ri $F(t)=\bigcup_{k=1}^{\infty} F_{k}(t)$ it follows that ri $F$ is also measurable.

REMARK. Since each $F_{k}$ has a measurable graph, the equality ri $F(t)=\bigcup_{k=1}^{\infty} F_{k}(t)$ implies that ri $F$ has a measurable graph. 
6. Lemmas concerning support functions. Let $A$ be a nonempty set in $S$. The support function $s(p, A)$ of $A$ is defined for every $p \in S$ by

$$
s(p, A)=\sup \{p \cdot x: x \in A\} .
$$

The reader can verify that we have already dealt with support functions. Thus, in $\S 3$ the function $p \rightarrow \nu_{p}(E)$ is the support function of $\Phi(E)$. In Lemma 5.1 the function $s(\cdot, t)$ is the support function of $F(t)$.

Let $A$ be a nonempty set in $S$. Denote $P(A)=\{p \in S: s(p, A)<\infty\}$. We shall see later that $P(A)$ is a convex cone. Denote $L(A)=P(A)-P(A)$, then $L(A)$ is a subspace, and $P(A)$ has full dimension in $L(A)$.

Let $(T, \mathscr{T}, \lambda)$ be a measure space. A set-valued measure $\Phi$ on $(T, \mathscr{T})$ is absolutely continuous with respect to $\lambda$ (or $\lambda$-continuous) if $\lambda(E)=0$ implies $\Phi(E)=\{0\}$. We denote this by $\Phi \ll \lambda$. It is clear that $\Phi \ll \lambda$ implies $\nu_{p} \ll \lambda$ for every $p \in S$.

LEMMA 6.1. The support function $s(\cdot, A)$ is a convex, lower semicontinuous and positively-homogeneous function from $S$ to $(-\infty, \infty]$. Conversely, if $s(p)$ is a convex function from $S$ to $(-\infty, \infty]$, which is positively-homogeneous and lower semicontinuous, then it is the support function of a certain closed and convex set $A$. Namely $A=\bigcap_{p \in S}\{x: p \cdot x \leqq s(p)\}$.

Proof. See Rockafellar [17, Theorem 13.2 and Corollary 13.2.1]. (For Rockafellar's notation see $[17$, p. 52].)

Since $s(\cdot, A)$ is a convex and positively-homogeneous function, it follows that $P(A)$ is a convex cone.

LEMMA 6.2. Let $s(\cdot)$ be the support function of a certain set. Let $p$ and $q$ be two vectors in $S$, and suppose $s(q)<\infty$. Define $q_{k}=(1 / k) q+(1-1 / k) p$ for $k=1,2, \ldots$ Then $s(p)=\lim s\left(q_{k}\right)$.

Proof. The convexity of $s$ implies $s\left(q_{k}\right) \leqq(1 / k) s(q)+(1-1 / k) s(p)$. The right side of the inequality converges to $s(p)$. Thus, $\lim \sup s\left(q_{k}\right) \leqq s(p)$. On the other hand, the lower semicontinuity of $s$ implies $\lim \inf s\left(q_{k}\right) \geqq s(p)$. Q.E.D.

Lemma 6.3. Let $A$ be a closed and convex set in $S$. Let $Q=\left\{q_{j}\right\}_{j=1}^{\infty}$ be a dense sequence in $L(A)$. Then $A=\bigcap_{j=1}^{\infty}\left\{x: q_{j} \cdot x \leqq s\left(q_{j}, A\right)\right\}$.

Proof. In view of Lemma 6.1, it is enough to show that the sequence of inequalities $q_{j} \cdot x \leqq s\left(q_{j}, A\right)$ implies that $p \cdot x \leqq s(p, A)$ for every $p \in P(A)$. Fix $q \in \operatorname{ri} P(A)$ and define $q_{k}=(1 / k) q+(1-1 / k) p$ for $k=1,2, \ldots$ Then $q_{k} \in \operatorname{ri} P(A)$ for every $k$ (see [17, Theorem 6.1]). According to Lemma 6.2, $s\left(q_{k}, A\right) \rightarrow s(p, A)$. Since $s(\cdot, A)$ is continuous in ri $P(A)$ [17, Theorem 10.1], there exists a subsequence $\left\{q_{l}\right\}_{l=1}^{\infty}$ of $Q$, for which $q_{l} \rightarrow p$ and $s\left(q_{l}, A\right) \rightarrow s(p, A)$. Indeed, $Q \cap P(A)$ is dense in $P(A)$, since $P(A)$ has full dimension in $L(A)$. Finally, notice that $q_{l} \cdot x \leqq s\left(q_{l}, A\right)$ for $l=1,2, \ldots$ implies $p \cdot x \leqq s(p, A)$. Q.E.D. 
LEMMA 6.4. Let $Q=\left\{q_{j}\right\}_{j=1}^{\infty}$ be a dense sequence in a relatively open cone $P$. Let $s(\cdot)$ be a function from $Q$ to $(-\infty, \infty)$. Suppose that the positively-homogeneous extension of $s$ is well defined and convex on the set $\{\alpha q: \alpha \geqq 0, q \in Q\}$. Then there exists a certain closed and convex set $A$, such that $s(q, A)=s(q)$ for $q \in Q$, and such that $P(A) \subset \operatorname{cl} P$. The set $A$ is given by $A=\bigcap_{j=1}^{\infty}\left\{x: q_{j} \cdot x \leqq s\left(q_{j}\right)\right\}$.

Proof. The idea of our proof is based on a proof of a similar theorem, due to Fenchel, where $Q$ is a convex and relatively open cone. Only a few modifications have to be done. Without loss of generality $P$ is an open cone. It is obvious that $s(q, A) \leqq s(q)$ for each $q \in Q$. Let $U$ be the set in the $S \oplus R$ space given by

$$
U=\operatorname{cl} \operatorname{co}\{(q, r): q \in Q, r \geqq s(q)\} .
$$

We claim that $q \in Q$ implies that $(q, s(q)) \in \partial U$. Otherwise, $(q, r) \in U$ for a certain $r<s(q)$. Also $(q, r)$ is a convex combination $\sum_{k=1}^{l} \alpha_{k}\left(q_{k}, r_{k}\right)$ where $r_{k} \geqq s\left(q_{k}\right)$ and $q_{k} \in Q$. Thus

$$
s(q)>r=\sum_{k=1}^{l} \alpha_{k} r_{k} \geqq \sum_{k=1}^{l} \alpha_{k} s\left(q_{k}\right)
$$

while $q=\sum_{k=1}^{l} \alpha_{k} q_{k}$. This is a contradiction to the convexity condition on $s$.

Thus $(q, s(q)) \in \partial U$, and there exists a support hyperplane $\left(u, u_{0}\right)$, to $U$ at $(q, s(q))$, i.e. $u \cdot p+u_{0} r \leqq u \cdot q+u_{0} s(q)$ for every $(p, r) \in U$. Without loss of generality $u_{0}=-1$. Indeed, since $P$ is open, $u_{0}<0$. Now, for every $(p, r) \in U$

$$
-r+u \cdot p \leqq-s(q)+u \cdot q
$$

Substitute $p=\alpha q$ in (1), and get

$$
(1-\alpha) s(q) \leqq(1-\alpha) u \cdot q .
$$

Use this for $\alpha<1$ and $\alpha>1$ in order to show that $s(q) \leqq u \cdot q$ and $s(q) \geqq u \cdot q$, respectively, thus $s(q)=u \cdot q$. Subtracting this from (1) implies $u \cdot p \leqq r$ for every $p \in Q$ and $r \geqq s(p)$. Thus, $u \cdot p \leqq s(p)$ for every $p \in Q$. Hence $u \in A$, and this together with the equality $s(q)=u \cdot q$ implies $s(q) \leqq s(q, A)$. Finally, it is easy to verify that $P(A)$ is a subset of the closed and convex cone spanned by $\{q \in Q: s(q)<\infty\}$. Q.E.D.

COROLlaRY 6.5. Let $Q=\left\{q_{j}\right\}_{j=1}^{\infty}$ be a dense sequence in a certain subspace $L$. Let $s(\cdot)$ be a function from $Q$ to $(-\infty, \infty]$. Suppose that the positively-homogeneous extension of $s$ is well defined, and convex on the set $\{\alpha q: \alpha \geqq 0, q \in Q\}$. Denote $P$ the convex cone spanned by $\{q \in Q: s(q)<\infty\}$. Then there exists a certain closed and convex set $A_{1}$, such that $s\left(q, A_{1}\right)=s(q)$ for every $q$ in $Q \cap$ ri $P$, and such that $P\left(A_{1}\right)$ $\subset \operatorname{cl} P$. The set $A_{1}$ is given by $A_{1}=\bigcap_{j=1}^{\infty}\left\{x: q_{j} \cdot x \leqq s\left(q_{j}\right)\right\}$.

Proof. By applying Lemma 6.4 to the sequence $Q \cap$ ri $P$ and to the relatively open cone ri $P$, we get a set $A$, such that $P(A) \subset \operatorname{cl} P$ and $s(q, A)=s(q)$ for every $q$ in $Q \cap$ ri $P$. We claim that $A=A_{1}$. In order to see this it is enough to show that the set of inequalities $q \cdot x \leqq s(q, A)$ for $q \in$ ri $P$ implies that $q \cdot x \leqq s(q)$ for every 
$q \in Q \cap \operatorname{cl} P$. The function $s(q, A)$ is lower semicontinuous, and is equal to the convex function $s(q)$ on a dense sequence, thus $s(q, A)$ is less or equal to $s(q)$ for every $q$. Q.E.D.

Throughout the following three lemmas, $(T, \mathscr{T}, \lambda)$ is a measure space such that $\lambda$ is a finite and nonnegative measure.

LEMMA 6.6. Let $F(t)$ be a measurable set-valued function and let $s(q, t)$ $=s(q, F(t))$ be its support function. Suppose that for every $q$, the integral $\int_{T} s(q, t) d \lambda(t)$ exists, and is finite or $+\infty$. Let $A$ be a subset of $S$. Let $Q=\left\{q_{j}\right\}_{j=1}^{\infty}$ be $a$ dense sequence in $P(A)$. If $\int_{T} s(q, t) d \lambda(t)=s(q, A)$ for every $q \in Q$, then $\int_{T} s(q, A) d \lambda(t)=s(q, A)$ for each $q$ in $\operatorname{cl} P(A)$.

Proof. Notice that the measurability of $s(q, \cdot)$ follows from Lemma 5.1. The function $s(q, t)$ is a convex function for each $t$, thus its integral $\int_{T} s(q, t) d \lambda(t)$ is a convex function, which is finite on a dense subset of $P(A)$, thus it is finite and continuous at least in ri $P(A)$; hence, $\int_{T} s(q, t) d \lambda(t)=s(q, A)$ for every $q \in \operatorname{ri} P(A)$. Let $p \in P(A)$ and $q \in \operatorname{ri} P(A)$, and define $q_{k}=(1 / k) q+(1-1 / k) p$, for $k=1,2, \ldots$ According to Lemma $6.2, s\left(q_{k}, A\right) \rightarrow s(q, A)$. We show now that

$$
s\left(q_{k}, A\right)=\int_{T} s\left(q_{k}, t\right) d \lambda(t) \rightarrow \int_{T} s(q, t) d \lambda(t)
$$

and this will complete the proof. The convexity of $\int_{T} s(q, t) d \lambda(t)$ implies

$$
\int_{T} s(q, t) d \lambda(t) \geqq \limsup _{k} \int_{T} s\left(q_{k}, t\right) d \lambda(t) .
$$

According to Lemma 6.2, $s\left(q_{k}, t\right) \rightarrow s(q, t)$ for every $t$; thus Fatou's Lemma implies

$$
\int_{T} s(q, t) d \lambda(t) \leqq \liminf _{k} \int_{T} s\left(q_{k}, t\right) d \lambda(t) . \quad \text { Q.E.D. }
$$

LemMA 6.7. Let $\Phi$ be a set-valued measure such that $\Phi \ll \lambda$. Suppose that there exists a certain subspace $M$ such that $L(\Phi(E))=M$ for every $E \in \mathscr{T}$ with a positive $\lambda$-measure. Then there exists a measurable set-valued function $F$, with convex and closed values, such that if $s(q, t)$ is the support function of $F(t)$ then for every $E \in \mathscr{T}$ and every $p \in S$ it is true that

$$
\int_{E} s(p, t) d \lambda(t)=\nu_{p}(E) .
$$

Proof. Let $Q=\left\{q_{j}\right\}_{j=1}^{\infty}$ be a dense sequence in $M$ and suppose that $Q$ has the structure of the vectors with rational components, i.e., if $q$ and $p$ are in $Q$ and $\alpha$ is rational, then $\alpha p+(1-\alpha) q$ is in $Q$. For each $q$ in $Q$, the measure $v_{q}$ is absolutely continuous with respect to $\lambda$. Let $g(q, t)$ be a Radon-Nikodym derivative, i.e. $\int_{E} g(q, t) d \lambda(t)=v_{q}(E)$ for every $E$ in $\mathscr{T}$. In particular, $g(q, t)>-\infty$ for $\lambda$-almost every $t$. The following two properties hold $\lambda$-almost everywhere. 
(a) The positively-homogeneous extension of $g(q, t)$ is well defined and convex on the set $\{\alpha q: \alpha \geqq 0, q \in Q\}$.

(b) If $S(t)=\{q: g(q, t)<\infty\}$ then $S(t)+S(t)=S(t)$, and $\alpha S(t)=S(t)$ for every positive rational number $\alpha$. Also the linear space spanned by $S(t)$ is $M$.

The proof of (a) and (b) is as follows. The function $q \rightarrow v_{q}(E)$ is positively homogeneous. This implies that $g(\alpha q, t)=\alpha g(q, t)$ for $\alpha \geqq 0$ and $q \in Q$ is well defined. The convexity of $v_{q}(E)=s(q, \Phi(E))$ implies the convexity of $g(q, t)$. Indeed, if $g(q, t)>\sum_{k=1}^{l} \alpha_{k} g\left(q_{k}, t\right)$ on a set with a positive $\lambda$-measure, then for a certain $E \in \mathscr{T}$

$$
\nu_{q}(E)=\int_{E} g(q, t) d \lambda(t)>\sum_{k=1}^{l} \alpha_{k} \int_{E} g\left(q_{k}, t\right) d \lambda(t)=\sum_{k=1}^{l} \alpha_{k} \nu_{q_{k}}(E),
$$

and if $q=\sum \alpha_{k} q_{k}$ is a convex combination, this leads to a contradiction. It is clear that $S(t)+S(t)=S(t)$ and that $\alpha S(t)=S(t)$ for a positive rational number $\alpha$. Finally, notice that $\lambda$-a.e. the set $S(t)$ includes $Q \cap P(\Phi(T))$. With this we finished the proof of (a) and (b).

We claim that $S(t)$ is a measurable set-valued function. Indeed if $C$ is a closed subset of $S$ then the set

$$
\{t: S(t) \cap C \neq \varnothing\}=\bigcup_{q \in Q \cap C}\{t: g(q, t)<\infty\}
$$

is obviously measurable. Denote by $P(t)$ the closed and convex cone spanned by $S(t)$. Then $S(t)$ is dense in $P(t)$, and according to Lemma 5.2 the set-valued function $t \rightarrow P(t)$ is measurable.

Without loss of generality all the above properties and discussion are valid for every $t$. For each $t$ the function $g(\cdot, t)$ satisfies the conditions of Corollary 6.5; hence, let $F(t)=\bigcap_{q \in Q}\{x: q \cdot x \leqq g(q, t)\}$ be the closed and convex set derived from this corollary. We shall show that $F(t)$ is the required set-valued function. In view of Lemma 5.5(c), the set-valued function $F$ is measurable. Let $E \in \mathscr{T}$ be with positive $\lambda$-measure. Then $\lambda$-a.e. in $E$, the set $P(t)$ includes $P(\Phi(E)$ ). Thus (see Corollary 6.5) $s(q, t)=g(q, t)$ for every $q \in$ ri $P(\Phi(E))$. Hence,

$$
\int_{E} s(q, t) d \lambda(t)=\int_{E} g(q, t) d \lambda(t)=\nu_{q}(E)
$$

for every $q \in$ ri $P(\Phi(E))$ and, according to Lemma 6.6, the equality holds also for every $q \in \operatorname{cl} P(\Phi(E))$. The next step is to verify the equality for every $p \notin \operatorname{cl} P(\Phi(E))$. We have to show that if $p \notin \operatorname{cl} P(\Phi(E))$ then $\int_{E} s(p, t) d \lambda(t)=\infty$. Denote $E_{1}$ $=\{t \in E: p \in P(t)\}$. According to Lemma 5.4 the set $E_{1}$ is measurable. On $E \backslash E_{1}$

$$
\int_{E \mid E_{1}} s(p, t) d \lambda(t)=\int_{E \backslash E_{1}} g(p, t) d \lambda(t)=\nu_{p}\left(E \backslash E_{1}\right)
$$


and if $\lambda\left(E_{1}\right)=0$ we are finished. If $\lambda\left(E_{1}\right)>0$ we have to analyse $E_{1}$ separately. Fix $q \in$ ri $P(\Phi(E))$, and define $q_{k}=(1 / k) q+(1-1 / k) p$. Then $q_{k} \in \operatorname{ri} P(t)$ for $t \in E_{1}$. Thus for every $k$

$$
\int_{E_{1}} s\left(q_{k}, t\right) d \lambda(t)=\int_{E_{1}} g\left(q_{k}, t\right) d \lambda(t)=\nu_{q_{k}}\left(E_{1}\right) .
$$

Since $s\left(q_{k}, t\right) \rightarrow s(p, t)$ for every $t \in E_{1}$, it follows from Fatou's Lemma that

$$
\int_{E_{1}} s(p, t) d \lambda(t) \leqq \liminf _{k} \int_{E_{1}} s\left(q_{k}, t\right) d \lambda(t)=\lim _{k} v_{q_{k}}\left(E_{1}\right) .
$$

Since $s(p, t)$ is convex for every $t \in E_{1}$ it follows that

$$
\int_{E_{1}} s(p, t) d \lambda(t) \geqq \lim _{k} \inf \int_{E_{1}} s\left(q_{k}, t\right) d \lambda(t)=\lim _{k} \nu_{q_{k}}\left(E_{1}\right) .
$$

Thus

$$
\int_{E_{1}} s(p, t) d \lambda(t)=\lim _{k} \nu_{q_{k}}\left(E_{1}\right)=\nu_{p}(E)
$$

Since the required equality holds for $E_{1}$ and $E \backslash E_{1}$ it holds also for $E$. Q.E.D.

LEMma 6.8. Let $\Phi$ be a set-valued measure such that $\Phi \ll \lambda$. Then there is a measurable set-valued function $F$ with convex and closed values, such that if $s(p, t)$ is the support function of $F(t)$, then for every $E \in \mathscr{T}$ and $p \in S$ it is true that $\int_{E} s(p, t) d \lambda(t)=\nu_{p}(E)$.

Proof. Note that the present lemma generalizes Lemma 6.7 by dropping the requirement that $M$ exists. The proof is to reduce the general case to the former.

Notice that if $E_{1} \subset E_{2}$ then $L\left(\Phi\left(E_{1}\right)\right) \supset L\left(\Phi\left(E_{2}\right)\right)$. Also, Lemma 6.7 will still hold if we replace the condition $L(\Phi(E))=M$ for every $E \subset T$ with positive $\lambda$-measure by the requirement that $T=\bigcup_{j=1}^{\infty} T_{j}$ and, for each $j$, the equality $L(\Phi(E))=M$ holds for every $E \subset T_{j}$ with a positive $\lambda$-measure.

Let $n_{0}=\max \operatorname{rank} L(\Phi(E))$, where the maximum is over the sets $E$ with a positive $\lambda$-measure. Let $\left(L_{\alpha}\right)_{\alpha}$ be the subspaces of rank $n_{0}$, for which there exists an $E_{\alpha}$, with $\lambda\left(E_{\alpha}\right)>0$, and such that $L_{\alpha}=L\left(E_{\alpha}\right)$. Notice that if $E \subset E_{\alpha}$ and $\lambda(E)>0$ then $L(\Phi(E))=L_{\alpha}$. This follows from the maximality of $n_{0}$. We show that $L_{\alpha} \neq L_{\beta}$ implies $\lambda\left(E_{\alpha} \cap E_{\beta}\right)=0$. Indeed, $L\left(\Phi\left(E_{\alpha} \cap E_{\beta}\right)\right)$ includes both $L_{\alpha}$ and $L_{\beta}$ and thus it has strictly greater rank. Hence, the family $\left(L_{\alpha}\right)_{\alpha}$ is countable or finite. Applying the standard exhausting procedure in order to define for each $\alpha$ a set $T_{\alpha}$ which is a countable union of sets $E_{j}$, for which $L\left(\Phi\left(E_{j}\right)\right)=L_{\alpha}$, and $T_{\alpha}$ is maximal in the sense that $L(\Phi(E))=L_{\alpha}$, implies $\lambda\left(E \backslash T_{\alpha}\right)=0$. It is clear that $\left(T_{\alpha}\right)_{\alpha}$ is a countable family of disjoint measurable sets, and as was pointed out before, the lemma holds for each $\left(T_{\alpha}, \mathscr{T}\left|T_{\alpha}, \lambda\right| T_{\alpha}\right)$.

Denote $T^{\prime}=T \backslash \bigcup_{\alpha} T_{\alpha}$. The set $T^{\prime}$ is measurable and $n_{1}=\max \operatorname{rank} L(\Phi(E))$ is strictly less than $n_{0}$, provided the maximum is taken over all the sets $E \subset T^{\prime}$ with 
$\lambda(E)>0$. By applying, for instance, an induction process (or by doing the above analysis for $T^{\prime}$, and by completing the procedure after a finite number of steps) we have decomposed $T$ into a countable number of measurable sets $T_{j}$, and the lemma holds for each $\left(T_{j}, \mathscr{T}\left|T_{j}, \lambda\right| T_{j}\right)$. Finally, the $\sigma$-additivity of each $\nu_{p}$ completes the proof. Q.E.D.

7. A lemma concerning integration of set-valued functions. Let $(T, \mathscr{T}, \lambda)$ be a measure space, where $\lambda$ is a finite and nonnegative measure. Let $F$ be a set-valued function from $T$ to the subsets of $S$, and suppose that the set $\{t: F(t)=\varnothing\}$ is $\lambda$ null. An integrable selection (with respect to $\lambda$ ) of $F$ is a $\lambda$-integrable function such that $f(t) \in F(t) \lambda$-a.e. The integral of $F$ over a set $E$ is defined by

$$
\int_{E} F(t) d \lambda(t)=\left\{\int_{E} f(t) d \lambda(t): f \mid E \text { is an integrable selection of } F\right\} .
$$

For simplicity, we write $\int_{E} F$ and $\int_{E} f$ instead of $\int_{E} F(t) d \lambda(t)$ and $\int_{E} f(t) d \lambda(t)$ respectively.

LEMMA 7.1. Let $\Phi$ be a set-valued measure on $(T, \mathscr{T})$ such that $\Phi$ takes on only convex values and such that $\Phi \ll \lambda$. Let $F(t)$ be the set-valued function originating in Lemma 6.8. Denote $G(t)=\mathrm{ri} F(t)$, for every $t \in T$. Then for every $E \in \mathscr{T}$ it is true that $\int_{E} G(t) d \lambda(t)=\operatorname{ri} \Phi(E)$.

Proof. It is enough to prove the lemma for $E=T$. The set-valued function $G$ has convex values, thus $\int_{T} G$ is a convex set. First we show that $\int_{T} G$ is included in ri $\Phi(E)$. Let $g$ be an integrable selection of $G$, and suppose that $y=\int_{T} g$ is not in ri $\Phi(T)$. Then there is a vector $p \in S$ such that

$$
p \cdot y \geqq \nu_{p}(T)>-\nu_{-p}(T) .
$$

Since $\lambda$-almost everywhere $p \cdot g(t) \leqq s(p, t)$, and since

$$
\int_{T} p \cdot g(t)=\int_{T} s(p, t) d \lambda(t)=\nu_{p}(T)
$$

it follows that $p \cdot g(t)=s(p, t)$ for a.e. $t$. Since

$$
\int_{T} s(p, t) d \lambda(t)=\nu_{p}(T)>-\nu_{-p}(T)=-\int_{T} s(-p, t) d \lambda(t)
$$

it follows that $s(p, t)>-s(-p, t)$ on a set $E$ with positive $\lambda$-measure. For $t \in E$ the equality $p \cdot g(t)=s(p, t)$ implies that $g(t) \notin$ ri $F(t)$, a contradiction.

In order to prove the inverse inclusion we show that $s\left(p, \int_{T} G\right) \geqq \nu_{p}(T)$ for every $p$ in $S$. Then the closure of $\int G$ includes the closure of $\Phi(T)$, and since both are convex it follows that $\int G$ includes ri $\Phi(T)$. We show that $s\left(p, \int_{T} G\right) \geqq \nu_{p}(T)$ by constructing (for every $p$ ) a sequence $g_{k}$ of integrable selections of $G$ such that $\int_{T} p \cdot g_{k} \rightarrow \nu_{p}(T)$. Let $x(t)$ be an integrable selection of $G$. Later we shall prove the existence of such a selection. Let us treat the following two cases separately. 
(i) The set $E=\{t: s(p, t)=\infty\}$ is null.

(ii) The set $E=\{t: s(p, t)=\infty\}$ has a positive $\lambda$-measure.

In case (i) define $F_{k}(t)=F(t) \cap\{x: p \cdot x \geqq s(p, t)-1 / k\}$. Then $F_{k}$ is a measurable set-valued function (Lemmas 5.3 and 5.5(a)) with closed and convex values, and thus (Lemma 5.6) ri $F_{k}$ has a measurable selection $h_{k}$. Notice that ri $F_{k}(t) \subset$ ri $F(t)$; thus, $h_{k}$ is a selection of $G$. Finally, define

$$
\begin{aligned}
g_{k}(t) & =h_{k}(t), & & \left\|h_{k}(t)\right\| \leqq k, \\
& =x(t) & & \text { otherwise, }
\end{aligned}
$$

then it is easy to verify that $\int_{T} p \cdot g_{k} \rightarrow \int_{T} s(p, t)=\nu_{p}(T)$.

In case (ii) define $F_{k}(t)=F(t) \cap\{x: p \cdot x \geqq k\}$, for $t \in E$. Then $F_{k}$ is measurable, and ri $F_{k} \subset$ ri $F$ has a selection $h_{k}$. For $m$ large enough, the function

$$
\begin{aligned}
g_{k} & =h_{k}(t), & & \left\|h_{k}(t)\right\| \leqq m, \\
& =x(t), & & \text { otherwise, }
\end{aligned}
$$

is an integrable selection of $G$, and

$$
\int p \cdot g_{k} \geqq k \lambda(E)-1-\int|p \cdot x| .
$$

Thus, $\int p \cdot g_{k} \rightarrow \infty=\nu_{p}(T)$.

Finally, as was promised before, we show that $G$ has an integrable selection $x$. Let $e_{1}, \ldots, e_{2 n}$ be the $2 n$ vectors $(0, \ldots, 0, \pm 1,0, \ldots, 0)$. We shall construct successively $2 n$ set-valued functions, $F_{1}, \ldots, F_{2 n}$, such that $F_{2 n}$ will be measurable, with convex and closed values and such that ri $F_{2 n} \subset F$. Also the functions $s\left(e_{i}, F_{2 n}\right)$ will be integrable for $i=1, \ldots, 2 n$; thus, any measurable selection of ri $F_{2 n}$ will be integrable, and according to Lemma 5.6 there exists a measurable selection. The construction of $F_{1}$ is as follows. The function $s\left(-e_{1}, t\right)$ is measurable and $\int s\left(-e_{1}, t\right)>-\infty$. Let $A=\left\{t: s\left(-e_{1}, t\right)>0\right\}$ and define

$$
\begin{aligned}
F_{1}(t) & =F(t) \cap\left\{x: e_{1} \cdot x \leqq 0\right\}, & & t \in A, \\
& =F(t) \cap\left\{x: e_{1} \cdot x \leqq-s\left(-e_{1}, t\right)+1\right\}, & & t \notin A .
\end{aligned}
$$

Then it is clear that $F_{1}$ is measurable, with convex and closed values, ri $F_{1} \subset$ ri $F$ and if $s_{1}(q, t)$ is the support function of $F_{1}$ then

$$
\int_{T} s_{1}\left(e_{1}, t\right)=\int_{A} s_{1}\left(e_{1}, t\right)+\int_{T \backslash A} s_{1}\left(e_{1}, t\right) \leqq 0+\int_{T \backslash A}\left(-s_{1}\left(-e_{1}, t\right)+1\right)<\infty .
$$

The construction of $F_{2}$ is the same, but replacing $F$ by $F_{1}, e_{1}$ by $e_{2}$ and $s$ by $s_{1}$. After a finite number of steps the construction of $F_{2 n}$ will be completed. Q.E.D.

The following corollary can be regarded as the integral generalization of the similar theorem which deals with sums (see [17, Corollary 6.6.2]).

COROLlary. Let $F$ be a measurable set-valued function with convex values, then $\int \operatorname{ri} F=\operatorname{ri} \int F$. 
Proof. Consider the set-valued measure $\Phi(E)=\int_{E} F$ and apply the proof of Lemma 7.1. Q.E.D.

8. Selectors. Let $\Phi$ be a set-valued measure on $(T, \mathscr{T})$. A vector-valued measure $\mu$ on $(T, \mathscr{T})$ is a selector of $\Phi$ if $\mu(E) \in \Phi(E)$ for every $E \in \mathscr{T}$.

We investigate in this section the existence of selectors, and their properties. In particular, the following problem is interesting: Give conditions implying that for every $E \in \mathscr{T}$ and every $x \in \Phi(E)$ there exists a selector $\mu$ of $\Phi$ such that $\mu(E)=x$.

Recall that $\Phi$ is absolutely continuous with respect to the nonnegative measure $\lambda$ if $\lambda(E)=0$ implies $\Phi(E)=\{0\}$.

THEOREM 8.1. Let $(T, \mathscr{T}, \lambda)$ be a measure space, $\lambda$ is finite and nonnegative. Let $\Phi$ be a set-valued measure, with convex values, and such that $\Phi \ll \lambda$. Then for every $x \in \Phi(E)$ there is a selector $\mu$ of $\Phi$ such that $\mu(E)=x$.

Proof. It is enough to consider the case $E=T$. If $\Phi(T)$ is a singleton then $\Phi(E)$ $=\{\mu(E)\}$ is a singleton for every $E \in \mathscr{T}$, and $\mu$ is a measure. Thus $\mu$ is the required selector. Proceed by induction on the dimension of $\Phi(T)$, and suppose $\operatorname{dim} \Phi(T)$ $>0$. Let $x$ be in $\Phi(T)$. If $x \notin$ ri $\Phi(T)$ then for a certain $p \in S$

$$
p \cdot x=\sup \{p \cdot y: y \in \Phi(T)\}>\inf \{p \cdot y: y \in \Phi(T)\} \text {. }
$$

Thus the countably additive set-valued set-function $\Phi_{p}$ (see $\S 3$ ) is a set-valued measure. Indeed, since $x \in \Phi_{p}(T)$ it follows that $\Phi_{p}(E) \neq \varnothing$ for every $E$. Obviously, $\Phi_{p}(T)$ has dimension less than $\Phi(T)$. According to the induction hypothesis there is a selector $\mu$ of $\Phi_{p}$ such that $\mu(T)=x$. It is clear that $\mu$ is also a selector of $\Phi$.

If $x \in \operatorname{ri} \Phi(T)$, consider the set-valued function $G$ constructed in Lemma 7.1. Then $\int_{E} G(t) d \lambda(t)=$ ri $\Phi(E)$ for every $E \in \mathscr{T}$. In particular, there exists an integrable selection $g$ of $G$ such that $\int_{T} g(t) d \lambda(t)=x$. Define $\mu(E)=\int_{E} g(t) d \lambda(t)$. Then $\mu$ is the required selector. Q.E.D.

REMARK. Neither the convexity condition, nor the condition $\Phi \ll \lambda$, can be omitted. Let $(I, \mathscr{B}, \lambda)$ be the unit interval, with the Borel $\sigma$-field and the Lebesgue measure. Consider first Hildenbrand's example, introduced in $\S 4$. Then $\Phi(E)=\{0\}$ for every $\lambda$-null set, and $\Phi(E)=\{0,1,2, \ldots\}$ for $E$ with $\lambda(E)>0$. It is clear that $\Phi \ll \lambda$. If $\mu$ is a selector of $\Phi$, then $\mu \ll \lambda$ and thus $\mu$ is nonatomic. If also $\mu(I)=1$, then the whole interval $[0,1]$ is in the range of $\mu$, a contradiction. Thus the only selector of $\Phi$ is the measure which is identically zero. The second example is as follows. If $E$ is a denumerable set, define $\Phi(E)=\{0\}$. Otherwise, set $\Phi(E)=(0, \infty)$. For any finite measure $\mu$ on $(I, \mathscr{B})$ one can construct an uncountable, measurable, $\mu$-null set (something like the Cantor set). Thus $\Phi$ does not admit a selector at all.

In the presence of atoms, the convexity condition on the values of $\Phi$ can be weakened. A complete characterization in the case $\Phi \ll \lambda$ is as follows.

THEOREM 8.2. Let $(T, \mathscr{T}, \lambda)$ be a measure space, where $\lambda$ is finite and nonnegative. Let $\Phi$ be a set-valued measure such that $\Phi \ll \lambda$. A necessary and sufficient condition, that for every $E$ and $x \in \Phi(E)$ there exists a selector $\mu$ of $\Phi$ such that $\mu(E)=x$, is that $\Phi$, restricted to the nonatomic part of $\lambda$, has only convex values. 
Proof. Since the measure $\lambda$ is finite, it has only $\boldsymbol{\aleph}_{0}$ atoms. Thus the expressions, atomic part and nonatomic part, of $\lambda$, have meaning. It is clear that we can treat separately each of these parts. Let $E_{1}, E_{2}, \ldots$ be a finite or countable collection of disjoint atoms of $\lambda$, and let $E=\bigcup_{i} E_{i}$. If $x \in \Phi(E)$, then $x=\sum_{i} x_{i}$, where $x_{i} \in \Phi\left(E_{i}\right)$. Define a measure $\mu$ on $E$ as follows. For each $i$ define $\mu\left(E_{i}\right)=x_{i}$, and let $E_{i}$ be an atom of $\mu$, or $x_{i}=0$. Then $\mu$ is a selector of $\Phi$. This proves the "sufficient" for the atomic part of $\lambda$. The "sufficient" for the nonatomic part follows from Theorem 8.1.

In order to prove the "necessary" for the nonatomic part let $x$ and $y$ be in $\Phi(E)$ and let $\theta$ satisfy $0<\theta<1$. Let $\mu_{1}$ and $\mu_{2}$ be two selectors of $\Phi$ such that $\mu_{1}(E)=x$ and $\mu_{2}(E)=y$. Since $\mu_{1} \ll \lambda$ and $\mu_{2} \ll \lambda$, it follows that both are nonatomic. Consider the $2 n$-dimensional vector-valued measure $\left(\mu_{1}, \mu_{2}\right)$. According to Lyapunov's convexity theorem there exists a subset $E^{\prime}$ of $E$ such that $\mu_{1}\left(E^{\prime}\right)$ $=\theta \mu_{1}(E)=\theta x$, and $\mu_{2}\left(E^{\prime}\right)=\theta \mu_{2}(E)=\theta y$. Since $\mu_{2}\left(E \backslash E^{\prime}\right)=(1-\theta) y$ it follows that

$$
\theta x+(1-\theta) y \in \Phi\left(E^{\prime}\right)+\Phi\left(E \backslash E^{\prime}\right)=\Phi(E) .
$$

Thus $\Phi(E)$ is convex. Q.E.D.

THEOREM 8.3. Let $(T, \mathscr{T})$ be a measurable space, and let $\Phi$ be a bounded setvalued measure on it. Then for every $E \in \mathscr{T}$ and $x \in \Phi(E)$, there is a selector $\mu$ of $\Phi$ such that $\mu(E)=x$.

Proof. Consider the measure $\nu$ defined in $\$ 4$. Then $v$ is finite and nonnegative, and $\Phi \ll \nu$. As was noted above, $\Phi$ is nonatomic on the nonatomic part of $\nu$. According to Theorem 4.2 the values of $\Phi$, on the nonatomic part of $v$, are convex. Thus, the sufficient conditions of Theorem 8.3 are fulfilled. Q.E.D.

We complete this section with some remarks on the atomic properties of the set-valued measures, and their selections. Recall that $\Phi$ is nonatomic if for every $E$ with $\Phi(E) \neq\{0\}$, there exists $E^{\prime} \subset E$ such that $\Phi\left(E^{\prime}\right) \neq\{0\}$ and $\Phi\left(E \backslash E^{\prime}\right) \neq\{0\}$. If $\Phi \ll \lambda$ and $\lambda$ is a finite, nonatomic measure then $\Phi$ is nonatomic. Indeed, suppose that $E$ is an atom of $\Phi$. Denote $\delta=\lambda(E)$, then $\delta>0$. There exists $E_{1} \subset E$ such that $\lambda\left(E_{1}\right)=2^{-1} \delta$ and $\Phi\left(E_{1}\right)=\{0\}$. There exists $E_{2} \subset\left(E \backslash E_{1}\right)$ such that $\lambda\left(E_{2}\right)=2^{-2} \delta$ and $\Phi\left(E_{2}\right)=\{0\}$. Proceed by induction, there exists $E_{k+1} \subset\left(E \backslash \bigcup_{l=1}^{k} E_{l}\right)$ such that $\lambda\left(E_{k+1}\right)=2^{-k-1} \delta$ and $\Phi\left(E_{k+1}\right)=\{0\}$. Finally denote $E_{0}=E \backslash \bigcup_{k=1}^{\infty} E_{k}$. Then $\lambda\left(E_{0}\right)=0$ and since $\Phi \ll \lambda$ it follows that $\Phi\left(E_{0}\right)=\{0\}$. This implies that $\Phi(E)$ $=\sum_{k=0}^{\infty} \Phi\left(E_{k}\right)=\{0\}$, a contradiction.

If $\mu$ is a selector of $\Phi$, and $\Phi \ll \lambda$ where $\lambda$ is nonatomic then $\mu \ll \lambda$, thus also $\mu$ is nonatomic. The following example shows that a selector of a nonatomic setvalued measure might be atomic. Let $T$ be $[0,1]^{\aleph}$ and let $\mathscr{T}$ be the Baire $\sigma$-field, i.e. the $\sigma$-field generated by the compact $G_{\delta}$ subsets of $T$. Define $\Phi(E)=[0, \infty)$ for every nonempty $E$ in $\mathscr{T}$. Then $\Phi$ is a nonatomic set-valued measure. Let $t \in T$ and define $\mu(E)=1$ if $t \in E$, and $\mu(E)=0$ otherwise. Obviously, $\mu$ is a purely atomic selection of $\Phi$. 
9. The derivative of a set-valued measure. Let $(T, \mathscr{T}, \lambda)$ be a finite, nonnegative measure space, and let $\Phi$ be a set-valued measure on $(T, \mathscr{T})$ such that $\Phi \ll \lambda$. The set-valued function $F$, from $T$ to the subsets of $S$, is a Radon-Nikodym derivative of $\Phi$ (with respect to $\lambda$ ) if

$$
\int_{E} F(t) d \lambda(t)=\Phi(E) \text { for every } E \in \mathscr{T} .
$$

A general theorem on the existence of derivatives will be introduced in the next section. Here we give two theorems concerning set-valued measures which have derivatives with convex and closed values and, respectively, with convex and relatively open values. Following Debreu and Schmeidler [8], we use the following terms. For two set-valued measures, $\Psi_{1}$ and $\Psi_{2}$, we write $\Psi_{1} \subset \Psi_{2}$ if $\Psi_{1}(E) \subset \Psi_{2}(E)$ for every $E \in \mathscr{T}$. Let $\Phi$ be a fixed set-valued measure, with convex values. Denote by $\mathscr{M}$ the collection of all the set-valued measures $\Psi$ with convex values such that $\operatorname{cl} \Psi(E)=\operatorname{cl} \Phi(E)$ for every $E$. (Notice the difference between Debreu-Schmeidler notation, and ours. They required only that $\Psi(E) \subset \mathrm{cl} \Phi(E)$.) Denote by $\Phi$ and $\check{\Phi}$, respectively, the greatest and smallest elements in $\mathscr{M}$, with respect to the order $\subset$, if such elements exist.

The following theorem was proved by Debreu and Schmeidler [8], for the case $\Phi$ takes on values in the nonnegative orthant. (They have actually proved more; see the remark which follows the theorem.)

THEOREM 9.1. Let $(T, \mathscr{T}, \lambda)$ be a finite, nonnegative measure space. Let $\Phi$ be a set-valued measure with convex values, and $\Phi \ll \lambda$. Then $\mathscr{M}$ has a greatest element $\Phi$, and $\Phi$ has a measurable Radon-Nikodym derivative, with closed and convex values.

Proof. Let $F$ be the set-valued function, constructed in Lemma 6.8. Then $F$ is measurable, with closed and convex values. It is clear that $\int_{E} F$ is included in $\operatorname{cl} \Phi(E)$ for every $E \in \mathscr{T}$. On the other hand, Lemma 7.1 implies that $\mathrm{ri} \Phi(E)$ is included in $\int_{E} F$. Thus the set-valued measure

$$
\Phi(E)=\int_{E} F(t) d \lambda(t)
$$

is in $\mathscr{M}$. We show that $\Phi$ is the greatest element in $\mathscr{M}$. Let $\Psi$ be in $\mathscr{M}$ and let $x \in \Psi(E)$. According to Theorem 8.1 there is a selector $\mu$ of $\Psi$ such that $\mu(E)=x$. Let $g(t)$ be the Radon-Nikodym derivative of $\mu$ with respect to $\lambda$. Then for every $p \in S$ and $E^{\prime} \in \mathscr{T}$

$$
p \cdot \int_{E^{\prime}} g(t) d \lambda(t)=p \cdot \mu\left(E^{\prime}\right) \leqq \nu_{p}\left(E^{\prime}\right) .
$$

This implies that $p \cdot g(t) \leqq s(p, t)$ for almost every $t$. The next step is to show that $g(t) \in F(t) \lambda$-a.e. Let $T_{j}$ for $j=1,2, \ldots$ be the decomposition of $T$ into disjoint sets obtained in Lemma 6.8. For every $T_{j}$ there exists a sequence $Q=\left\{p_{k}\right\}_{k=1}^{\infty}$ such 
that for almost every $t$ in $T_{j}$

$$
F(t)=\bigcap_{k=1}^{\infty}\left\{x: p_{k} \cdot x \leqq s\left(p_{k}, t\right)\right\} .
$$

Since $p_{k} \cdot g(t) \leqq s\left(p_{k}, t\right)$ holds for every $p_{k}$, almost everywhere, it follows that almost everywhere $p_{k} \cdot g(t) \leqq s\left(p_{k}, t\right)$ for every $p_{k}$. Thus almost everywhere in $T_{j}$ it is true that $g(t) \in F(t)$. Finally, since $g$ is an integrable selection of $F$, it follows that $x=\int_{E} g(t) d \lambda(t)$ belongs to $\Phi(E)$. Q.E.D.

REMARK. Theorem 9.1 shows that if $\Phi$ is the greatest element in $\mathscr{M}$ then it has a derivative with closed and convex values. Is the "only if" part true? The answer in the general case is negative. The answer is positive if $\Phi$ has values in the positive orthant. This was proved by Debreu and Schmeidler in [8]. The proof is based on the following fact (see [8, Lemma 7]). If $\Phi$ is defined by $\Phi(E)=\int_{E} H$, where $H$ is measurable, and its values are closed convex subsets of the nonnegative orthant, then a.e. $F(t) \subset H(t)$. (If $H$ is not measurable, one can show that every integrable selection of $F$ is a selection of $H$.) Thus

$$
\Phi(E)=\int_{E} F \subset \int_{E} H=\Phi(E) .
$$

Hence $\Phi=\Phi$. If $H$ might have values not in the nonnegative orthant, the situation is different. Consider the following example.

Let $T=[0,1]$ and define $H(t)$ in the two-dimensional plane by

$$
H(t)=\{(x, t x):-\infty<x<\infty\} .
$$

It is easy to see that if $\lambda(E)>0$ then $\int_{E} H=S$. Thus $F(t)$ is identically $S$, and obviously it is never true that $F(t) \subset H(t)$. However, in this example it is true that $\int_{E} H=\hat{\Phi}$, and this is what happens in all two-dimensional cases. In order to find a counterexample to the "only if" part we must pass to the three-dimensional space. Let $T=(0,1)$. Consider the sets

$$
\begin{aligned}
& A(t)=\left\{(x, t x, z): x>0, z \geqq x\left(1+t^{2}\right)^{1 / 2}\right\}, \\
& B(t)=\{(x,-(1 / t) x, 0):-\infty<x<\infty\},
\end{aligned}
$$

and define $H(t)=A(t) \oplus B(t)$. Then it is easy to see that if $\lambda(E)>0$ then

$$
\int_{E} H(t) d \lambda(t)=\Phi(E)=\{(x, y, z): z>0\} .
$$

Hence $\Phi(E)=\{(x, y, z): z \geqq 0\}$ strictly includes $\Phi(E)$.

THEOREM 9.2. Let $(T, \mathscr{T}, \lambda)$ be a finite and nonnegative measure space. Let $\Phi$ be a set-valued measure with convex values, and $\Phi \ll \lambda$. Then $\mathscr{M}$ has a smallest element $\check{\Phi}$, and $\check{\Phi}$ has a measurable Radon-Nikodym derivative with relatively open and convex values. 
Proof. Let $G=\operatorname{ri} F$, where $F$ is the set-valued function constructed in Lemma 6.8. Then $G$ has convex and relatively open values. In view of Lemma 7.1, for every $E \in \mathscr{T}$,

$$
\int_{E} G(t) d \lambda(t)=\operatorname{ri} \Phi(E)
$$

Thus ri $\Phi(E)$ is a set-valued measure, and hence the smallest element $\check{\Phi}$ in $\mathscr{M}$. Also $G$ is the required derivative for $\check{\Phi}$. Q.E.D.

REMARK. The "only if" part of Theorem 9.2 is also true. Indeed let $H(t)$ be measurable with convex and relatively open values and let $\Phi(E)=\int_{E} H$. It is easy to see that $H(t) \subset G(t)$ a.e. Thus

$$
\Phi(E)=\int_{E} H \subset \int_{E} G=\check{\Phi}(E)
$$

and $\Phi$ is the smallest element. Without the measurability condition on $H$ it might happen that $\Phi$ strictly includes $\check{\Phi}$. Consider the following example. Let $E_{k}$ for $k=1,2, \ldots$ be the standard decomposition of the unit interval into disjoint nonmeasurable sets with inner measure zero and outer measure one. For $t \in E_{k}$ define $H(t)=(0,1+1 / k)$. Then $\Phi(E)=(0, \lambda(E)]$ strictly includes $\check{\Phi}(E)=(0, \lambda(E))$.

As a final remark to this section, notice that in both Theorems 9.1 and 9.2, if $\Phi$ has values in the nonnegative orthant, then the derivatives obtained for $\Phi$ and for $\check{\Phi}$ have values a.e. in the nonnegative orthant. This is an immediate conclusion from the construction of $F$ in Lemmas 6.7 and 6.8.

10. The general theorem on derivatives. In this section we prove that every set-valued measure $\Phi$, with convex values, has a Radon-Nikodym derivative with respect to the finite nonnegative measure $\lambda$, provided $\Phi \ll \lambda$. The proof uses the Continuum Hypothesis. It will be interesting if one can provide a proof without $\mathrm{CH}$.

The convexity of $\Phi$ is needed only for the nonatomic part of the measure $\lambda$. If $\lambda$ is purely atomic then every set-valued measure $\Phi$, such that $\Phi \ll \lambda$, has a derivative with respect to $\lambda$. Just define $H(t)=\Phi(E) / \lambda(E)$ for every $t$ in the atom $E$. On the other hand, if $\lambda$ is nonatomic then the convexity of the values of $\Phi$ is necessary for the existence of a derivative. (See Theorem 8.2.)

In the sequel, $(T, \mathscr{T}, \lambda)$ is a finite nonnegative measure space, $\Phi$ is a set-valued measure with convex values and $\Phi \ll \lambda$. We shall use the set-valued function $F$, constructed in Lemma 6.8, and $G(t)=\mathrm{ri} F(t)$ is as in Lemma 7.1. The support function of $F(t)$ is $s(p, t)$, and remember that $\int_{E} s(p, t) d \lambda(t)=v_{p}(E)$ for every $E \in \mathscr{T}$. Let $p$ be in $S$. Denote $F_{p}(t)=\{x \in F(t): p \cdot x=s(p, t)\}$ and recall that $\Phi_{p}(E)=\left\{x \in \Phi(E): p \cdot x=\nu_{p}(E)\right\}$. Finally, denote by $M_{p}(t)$ the hyperplane $\{x: p \cdot x=s(p, t)\}$.

LEMMA 10.1. Suppose $\Phi_{p}(T) \neq \varnothing$ and let $H_{p}(t)$ be a Radon-Nikodym derivative of $\Phi_{p}$. Then $H_{p}(t) \cap F_{p}(t)$ is also a derivative of $\Phi_{p}$. 
Proof. It is clear that $\int_{E} H_{p} \cap F_{p}$ is included in $\int_{E} H_{p}$. Conversely, let $x \in \Phi_{p}(E)$. Let $h$ be an integrable selection of $H_{p}$. See the last paragraph in the proof of Theorem 9.1 in order to be sure that $h$ is a selection of $F$. The equality $\int_{E} s(p, t) d \lambda(t)$ $=v_{p}(E)$, together with $p \cdot h(t) \leqq s(p, t)$, a.e. imply that $h(t) \in F_{p}(t)$ a.e. Q.E.D.

TheOrem 10.2. Assume the Continuum Hypothesis. Let $(T, \mathscr{T}, \lambda)$ be a finite, nonnegative measure space, $\Phi$ a set-valued measure with convex values, and $\Phi \ll \lambda$. Then $\Phi$ has a Radon-Nikodym derivative with respect to $\lambda$.

Proof. If $\Phi(T)$ is a singleton, the problem reduces to the existence of a RadonNikodym derivative of a vector-valued measure. Proceed by induction on the maximal dimension of $\Phi(E)$, where $E \in \mathscr{T}$. It will be convenient to adopt an induction hypothesis also for set-valued measures which might take on the empty set as a value, and then to prove the induction step for a set-valued measure $\Phi$, such that $\Phi(T) \neq \varnothing$. A standard use of the exhausting procedure will complete the induction step also for $\Phi$ such that $\Phi(T)=\varnothing$. Notice that if $\Phi(T) \neq \varnothing$, then the maximal dimension of $\Phi(E)$ is obtained by $\Phi(T)$. Indeed, $\Phi(T)$ contains a translation of each $\Phi(E)$. Without loss of generality $\Phi(T)$ has full dimension in $S$. Otherwise $\Phi(T)$ is contained in a hyperplane $\left\{x: q \cdot x=v_{q}(T)\right\}$, for a certain $q$ where $\|q\|=1$. Then it suffices to consider the set-valued measure $\Psi(E)=\Phi(E)-\nu_{q}(E) q$, which is contained in a proper subspace.

Let $A$ be the vectors in the unit sphere. Then for every $p \in A$, the set-valued setfunction $\Phi_{p}$ is countably additive (Proposition 3.2), and $\operatorname{dim} \Phi_{p}(E)$ is strictly less than $\operatorname{dim} \Phi(T)$, for every $E \in \mathscr{T}$. According to the induction hypothesis $\Phi_{p}$ has a Radon-Nikodym derivative $H_{p}$, and without loss of generality (Lemma 10.1) $H_{p}(t) \subset F_{p}(t)$ for every $t \in T$.

Suppose that $A$ is well ordered, and in view of the Continuum Hypothesis every set $\{q: q<p\}$ is denumerable. For each $p \in A$, define a set-valued function $\tilde{H}_{p}$ as follows. If $p=p_{1}$ is the first element of $A$ define $\tilde{H}_{p}(t)=H_{p}(t)$. Otherwise, define

$$
\tilde{H}_{p}(t)=H_{p}(t) \backslash\left(H_{p}(t) \cap \bigcup_{q<p} M_{q}(t)\right) .
$$

Finally, define $H(t)=G(t) \cup \bigcup_{p} \tilde{H}_{p}(t)$. We claim that $H$ is a Radon-Nikodym derivative of $\Phi$.

We show first that $\int_{E} H$ includes $\Phi(E)$ for every $E \in \mathscr{T}$. If $x \in$ ri $\Phi(E)$ then already $x \in \int_{E} G$ (see Lemma 7.1). If $x \notin \operatorname{ri} \Phi(E)$ then for a certain $p$ in $A$ it holds that $x \in \Phi_{p}(E)$. Then there exists an integrable selection $h$ of $H_{p}$ for which $\int_{E} h=x$. Proceed by induction on $p$. If $p=p_{1}$ then $\tilde{H}_{p}=H_{p}$ and $h$ is actually a selection of $H$. If $p \neq p_{1}$ define a sequence of measurable subsets of $E$ as follows. For $q=p_{1}$ define $E_{q}=\left\{t \in E: h(t) \in M_{q}(t)\right\}$. For $q \neq p_{1}$ and $q \leqq p$ define

$$
E_{q}=\left\{t \in E: h(t) \in M_{q}(t)\right\} \backslash \bigcup_{r<q} E_{r} .
$$


Each $E_{q}$ is measurable. This follows from Lemma 5.4 and the fact that $M_{q}(t)$ $=\{x: q \cdot x=s(q, t)\}$ is a measurable set-valued function (Lemma 5.5(b)). Notice that $\int_{E_{q}} h$ belongs to $\Phi_{q}\left(E_{q}\right)$ for every $q \leqq p$. By the induction hypothesis, there is an integrable selection $\tilde{h}$ of $H_{q}$ defined for $t \in E_{q}$ such that $\int_{E_{q}} \tilde{h}=\int_{E_{q}} h$. The definition of $\tilde{H}_{p}$ and the care we have taken while defining $E_{q}$ imply that $\tilde{h}(t) \in \tilde{H}_{q}(t)$ for $t \in E_{q}$. Thus $h$ is an integrable selection of $H$ and $\int_{E} h=x$.

The next step is to show that $\int_{E} H$ is included in $\Phi(E)$. Let $h$ be an integrable selection of $H$ and denote $x=\int_{E} h$. It is clear that $x \in \operatorname{cl} \Phi(E)$. If $x \in \mathrm{ri} \Phi(E)$ then there is nothing else to show. Otherwise, there exists a $p \in S$ such that $x \in\left\{y: p \cdot y=v_{p}(E)\right\}$. In this case $h(t) \in M_{p}(t)$ almost everywhere in $E$. Define the sequence of sets $E_{q}$, for $q \leqq p$, as was done above. Then it follows that $h(t)$ $\in H(t) \cup G(t)$ for $t \in E_{q}$. It is easy to verify that $\{t: h(t) \in G(t)\}$ is a measurable set. Thus $\int_{E_{q}} h$ belongs to $\Phi_{q}\left(E_{q}\right)$ for $q \leqq p$. Finally, $\Phi$ is a set-valued measure and $\{q: q \leqq p\}$ is a denumerable set, thus $\int_{E} h$ belongs to $\Phi(E)$. Q.E.D.

ACKNowledgment. The author wishes to thank Professor R. J. Aumann for his kind help and supervision.

\section{REFERENCES}

1. R. J. Aumann, Integrals of set-valued functions, J. Math. Anal. Appl. 12 (1965), 1-12. MR 32 \#2543.

2. - Existence of competitive equilibria in markets with a continuum of traders, Econometrica 34 (1966), 1-17. MR 32 \#9026.

3. H. T. Banks and M. Q. Jacobs, A differential calculus for multifunctions, J. Math. Anal. Appl. 29 (1970), 246-272. MR 42 \#846.

4. T. F. Bridgland, Trajectory integrals of set valued functions, Pacific J. Math. 33 (1970), 43-68. MR 41 \#7061.

5. C. Castaing, Sur les multi-applications measurables, Rev. Française Informat. Recherche Opérationnelle 1 (1967), 3-34.

6. R. Datko, Measurability properties of set-valued mapping in a Banach space, SIAM J. Control 8 (1970), 226-238.

7. G. Debreu, Integration of correspondences, Proc. Fifth Berkeley Sympos. Math. Statist. and Probability (Berkeley, Calif., 1965/66), vol. II: Contributions to Probability Theory, part 1, Univ. California Press, Berkeley, Calif., 1967, pp. 351-372. MR 37 \#3835.

8. G. Debreu and D. Schmeidler, The Radon-Nikodym derivative of a correspondence, Proc. Sixth Berkeley Sympos. Math. Statist. and Probability (Berkeley, Calif., 1970) (to appear).

9. H. Halkin, Some further generalizations of a theorem of Lyapunov, Arch. Rational Mech. Anal. 17 (1964), 272-277. MR 30 \#4625.

10. P. R. Halmos, The range of a vector measure, Bull. Amer. Math. Soc. 54 (1948), 416-421. MR 9, 574.

11. H. Hermes, Calculus of set valued functions and control, J. Math. Mech. 18 (1968/69), 47-59. MR 38 \#298.

12. H. Hermes and J. P. Lasalle, Functional analysis and time optimal control, Academic Press, New York, 1969.

13. W. Hildenbrand, Pareto optimality for a measure space of economic agents, Int. Econ. Rev. 10 (1969), 363-372. 
14. C. J. Himmelberg and F. S. Van Vleck, Some selection theorems for measurable functions, Canad. J. Math. 21 (1969), 394-399. MR 38 \#4637.

15. M. Hukuhara, Intégration des applications mesurables dont la valeur est un compact convexe, Funkcial. Ekvac. 10 (1967), 205-223. MR 37 \#2092.

16. M. Q. Jacobs, On the approximation of integrals of multivalued functions, SIAM J. Control 7 (1969), 158-177. MR 40 \#7419.

17. R. T. Rockafellar, Convex analysis, Princeton Univ. Press, Princeton, N. J., 1970.

18. - Measurable dependence of convex sets and functions on parameters, J. Math. Anal. Appl. 28 (1969), 4-25. MR 40 \#288.

19. D. Schmeidler, "Convexity and compactness in countably additive correspondence" in Differential games and related topics, North-Holland, Amsterdam, 1971.

20. K. Vind, Edgeworth-allocations in an exchange economy with many traders, Int. Econ. Rev. 5 (1964), 165-177.

The Hebrew University of Jerusalem, Jerusalem, Israel 